\title{
Stroke and recurrent haemorrhage associated with antithrombotic treatment after gastrointestinal bleeding in patients with atrial fibrillation: nationwide cohort study
}

\author{
Laila Staerk, ${ }^{1,2}$ Gregory Y H Lip, ${ }^{2}$ Jonas B Olesen, ${ }^{1}$ Emil L Fosbøl, ${ }^{3}$ Jannik L Pallisgaard, \\ Anders N Bonde, ${ }^{1}$ Anna Gundlund, ${ }^{1}$ Tommi B Lindhardt, ${ }^{1}$ Morten L Hansen, ${ }^{3}$ Christian \\ Torp-Pedersen, ${ }^{4}$ Gunnar H Gislason $n^{1,5,6,7}$
}

1Department of Cardiology,

Copenhagen University Hospital

Herlev, and Gentofte,

Kildegaardsvej 28, 2900

Hellerup, Denmark

${ }^{2}$ University of Birmingham

Centre for Cardiovascular

Sciences, Birmingham City

Hospital, Birmingham UK

${ }^{3}$ Department of Cardiology,

Copenhagen University Hospital

Rigshospitalet, Copenhagen,

Denmark

${ }^{4}$ Institute of Health, Science and

Technology, Aalborg University,

Aalborg, Denmark

${ }^{5}$ Faculty of Health and Medical

Sciences, University of

Copenhagen, Copenhagen,

Denmark

${ }^{6}$ National Institute of Public Health, University of Southern

Denmark, Copenhagen,

Denmark

7Danish Heart Foundation,

Copenhagen, Denmark

Correspondence to: L Staerk

Lailastaerk@gmail.com

Additional material is published online only. To view please visit

the journal online (http://dx.doi. org/10.1136/bmj.h5876)

Cite this as: BMJ 2015;351:h5876 doi: $10.1136 /$ bmj.h5876

Accepted: 12 October 2015

\section{ABSTRACT}

STUDY QUESTION

What are the risks of all cause mortality, thromboembolism, major bleeding, and recurrent gastrointestinal bleeding associated with restarting antithrombotic treatment after gastrointestinal bleeding in patients with atrial fibrillation?

METHODS

This Danish cohort study (1996-2012) included all patients with atrial fibrillation discharged from hospital after gastrointestinal bleeding while receiving antithrombotic treatment. Restarted treatment regimens were single or combined antithrombotic drugs with oral anticoagulation and antiplatelets. Follow-up started 90 days after discharge to avoid confounding from use of previously prescribed drugs on discharge. Risks of all cause mortality, thromboembolism, major bleeding, and recurrent gastrointestinal bleeding were estimated with competing risks models and time dependent multiple Cox regression models.

\section{STUDY ANSWER AND LIMITATIONS}

4602 patients (mean age 78 years) were included. Within two years, 39.9\% (95\% confidence interval $38.4 \%$ to $41.3 \%, n=1745)$ of the patients had died, $12.0 \%$ (11.0\% to $13.0 \%, n=526)$ had experienced thromboembolism, $17.7 \%$ ( $16.5 \%$ to $18.8 \%, n=788)$ major bleeding, and $12.1 \%(11.1 \%$ to $13.1 \%, n=546)$ recurrent gastrointestinal bleeding. $27.1 \%(n=924)$ of patients did not resume antithrombotic treatment. Compared with non-resumption of treatment, a reduced risk of all cause mortality was found in association with restart of oral anticoagulation

\section{WHAT IS ALREADY KNOWN ON THIS TOPIC}

The gastrointestinal tract is the most common site of bleeding due to oral anticoagulant treatment among patients with atrial fibrillation

Data on whether to restart antithrombotic treatment or withhold treatment after gastrointestinal bleeding are scarce

\section{WHAT THIS STUDY ADDS}

In this study over a third of patients with atrial fibrillation discharged after gastrointestinal bleeding while taking antithrombotics died within two years Under a third did not resume antithrombotics after gastrointestinal bleeding Restarting single treatment with oral anticoagulation was associated with the lowest risk of all cause mortality and thromboembolism, and a relative safe use regarding risk of recurrent bleeding, compared with non-resumption of antithrombotic treatment

(hazard ratio $0.39,95 \%$ confidence interval 0.34 to 0.46$)$, an antiplatelet agent $(0.76,0.68$ to 0.86$)$, and oral anticoagulation plus an antiplatelet agent $(0.41,0.32$ to 0.52$)$, and a reduced risk of thromboembolism was found in association with restart of oral anticoagulation (0.41, 0.31 to 0.54$)$, an antiplatelet agent $(0.76,0.61$ to 0.95$)$, and oral anticoagulation plus an antiplatelet agent $(0.54$, 0.36 to 0.82 ). Restarting oral anticoagulation alone was the only regimen with an increased risk of major bleeding $(1.37,1.06$ to 1.77$)$ compared with nonresumption of treatment; however, the difference in risk of recurrent gastrointestinal bleeding was not significant between patients who restarted an antithrombotic treatment regimen and those who did not resume treatment.

\section{WHAT THIS STUDY ADDS}

Among patients with atrial fibrillation who experience gastrointestinal bleeding while receiving antithrombotic treatment; subsequent restart of oral anticoagulation alone was associated with better outcomes for all cause mortality and thromboembolism compared with patients who did not resume treatment. This was despite an increased longitudinal associated risk of bleeding. FUNDING, COMPETING INTERESTS, DATA SHARING This study was supported by a grant from BoehringerIngelheim. Competing interests are available in the full paper on bmj.com. The authors have no additional data to share.

\section{Introduction}

Patients with non-valvular atrial fibrillation and risk factors for thromboembolism require anticoagulant treatment to reduce the risk of stroke. ${ }^{12}$ The major complication with anticoagulant treatment is the increased risk of bleeding, ${ }^{3}$ particularly gastrointestinal bleeding. ${ }^{4-6}$

After patients have experienced gastrointestinal bleeding during antithrombotic treatment, their clinicians face the clinical dilemma of whether to restart treatment or not. Moreover, there are several treatment regimens for clinicians to choose from: single or combined treatment with a vitamin $\mathrm{K}$ antagonist, a non-vitamin $\mathrm{K}$ antagonist oral anticoagulant, aspirin (acetylsalicylic acid), and an adenosine diphosphate receptor antagonist (clopidogrel, prasugrel, or ticagrelor). ${ }^{278}$ It is important to clarify how clinicians should treat patients with atrial fibrillation after antithrombotic related gastrointestinal bleeding; however, data 
on this are scarce, and no randomised controlled trial has been performed to elucidate the clinical dilemma regarding risk of stroke versus risk of bleeding in these patients. ${ }^{5910}$

In a nationwide cohort study, we examined the risk of all cause mortality and admission to hospital or deaths due to thromboembolism, major bleeding, or recurrent gastrointestinal bleeding associated with restarting antithrombotic treatment after a gastrointestinal bleed in patients with atrial fibrillation.

\section{Methods}

In Denmark, all residents receive a unique and permanent civil registration number at birth or immigration that allows linkage between nationwide registries at an individual level. We obtained data from the following three sources. Firstly, the Danish national patient registry, which holds information on all hospital admissions since 1978 and at discharge; each hospital admission is coded with one primary and, if appropriate, one or more secondary diagnoses, in accordance with the International Classification of Diseases, the eighth revision until 1994 and the 10th revision thereafter. In addition, the registry keeps information on all procedures and surgical operations since 1996 (the Nordic Medical Statistics Committees classification of surgical procedures). ${ }^{11}$ Secondly, the Danish national prescription registry, which records all claimed drug prescriptions from pharmacies in Denmark since 1995.12 Finally, the Danish civil registration system, which contains information about an individual's vital status and cause of death. ${ }^{13}$

\section{Study population}

We included all patients with atrial fibrillation who experienced gastrointestinal bleeding while receiving single or combined antithrombotic treatment with a vitamin K antagonist, dabigatran, rivaroxaban, aspirin, clopidogrel, prasugrel, or ticagrelor. Patients were included on the day of discharge from a first time admission to hospital owing to gastrointestinal bleeding in the study period from 1 January 1996 to 31 December 2012. We referred to the day of admission as the inclusion event and the day of discharge as the inclusion day.

In registries, the positive predictive value of bleeding related hospital admission is between $89 \%$ and $99 \% 0^{14}$; that is, among all patients with a diagnosis code of bleeding, $89 \%$ to $99 \%$ were actually admitted to hospital with bleeding. Moreover, the agreement with regard to specific bleeding site suggests that misclassification is rare. ${ }^{14}$

To assemble a homogeneous study population with atrial fibrillation and treated with antithrombotics, we excluded patients aged less than 30 or more than 100 , patients with valvular heart disease, patients with total hip or knee replacement surgery up to eight weeks before the inclusion event, and patients with deep vein thrombosis or pulmonary embolism up to six months before the inclusion event.

\section{Antithrombotic treatment}

To assess the resumption of antithrombotic treatment after discharge from hospital for gastrointestinal bleeding, we defined a 90 days blanking period-that is, follow-up did not start until inclusion day plus 90 days. All patients who died or experienced a thromboembolic event, major bleeding, or recurrent gastrointestinal bleeding in the 90 days blanking period were excluded. We divided the remaining patients who survived without an event until baseline, into groups according to type of antithrombotic treatment regimen restarted in the blanking period. The restarted groups were single treatment with oral anticoagulation (vitamin K antagonist, dabigatran, or rivaroxaban), single treatment with an antiplatelet agent (aspirin or an adenosine diphosphate receptor antagonist (clopidogrel, prasugrel, or ticagrelor)), and dual or triple treatment with oral anticoagulation, aspirin, and an adenosine diphosphate receptor antagonist.

We chose a blanking period of 90 days since in Denmark the numbers of tablets in the largest packet size available for antithrombotics last for approximately three months. This was done to remove the uncertainty about whether patients initially after discharge used antithrombotic drugs from an old package purchased before the gastrointestinal bleed. We kept records on the day each prescription was dispensed, the package size, and dosage. By using this method, we calculated patients' time dependent antithrombotic use during follow-up, as done in previous studies. ${ }^{41516}$

\section{Concomitant medical treatment and comorbidities}

To identify concomitant medical treatment at the time of the inclusion event, we used prescriptions filled from 0 to 90 days before the admission for gastrointestinal bleeding. To identify concomitant medical treatment at baseline, we used prescriptions filled during the 90 days blanking period. We determined comorbidities and invasive and surgical procedures from diagnosis and procedure codes based on validated methods. ${ }^{17-20}$

We assessed stroke and bleeding risks with risk stratification schemes according to $\mathrm{CHADS}_{2}$ (congestive heart failure, hypertension, age $\geq 75$ years, diabetes mellitus, stroke or transient ischaemic attack (double weight)), $\mathrm{CHA}_{2} \mathrm{DS}_{2}$-VASc ( $\mathrm{CHADS}_{2}$ with vascular disease, age 65 to 74, and female sex added and double weight of age $\geq 75$ years), and HAS-BLED (hypertension, abnormal renal or liver function, stroke, previous bleeding, international normalised ratio (left out because data are unfilled), elderly (age $>65$ years), and drug (antiplatelet agents, non-steroidal anti-inflammatory drugs) or alcohol misuse) scores as in previous studies. ${ }^{1521} \mathrm{~A}$ low $\mathrm{CHADS}_{2}$ or $\mathrm{CHA}_{2} \mathrm{DS}_{2}{ }^{-}$ VASc score indicated a low risk of stroke, and a low HAS-BLED score indicated a low risk of bleeding. The predicted risk of stroke or bleeding increases when the scores increase. Supplementary table 1 lists all Anatomical Therapeutic Chemical Classification System codes, diagnosis codes, and invasive procedure codes used in the study. 
Outcomes and follow-up

The outcomes of interest were all cause mortality or admission to hospital or death due to thromboembolism, major bleeding, or recurrent gastrointestinal bleeding. We defined thromboembolism by diagnosis codes for ischaemic stroke, transient ischaemic attack, or systemic thromboembolism. Validation of the ischaemic stroke diagnosis showed that among all patients listed with ischaemic stroke in the Danish national patient registry, $97 \%$ to $100 \%$ truly had ischaemic stroke-that is, the positive predictive value was $97 \%$ to $100 \%$. The positive predictive value for transient ischaemic attack ranges from $57.9 \%$ to $68.4 \% .{ }^{19}$ Major bleeding was determined by diagnosis codes of intracranial bleeding, or severe bleeding from the respiratory, gastrointestinal, or urinary tract. ${ }^{14}$ We identified recurrent gastrointestinal bleeding by all diagnosis codes of gastrointestinal bleeding. ${ }^{14}$

Follow-up of patients was until either of the outcomes, death, five year follow-up, or 31 December 2012, whichever came first.

\section{Statistical analysis}

We present the inclusion day and baseline characteristics as numbers and percentages or as means and standard deviations where appropriate. For categorical data we used $\chi^{2}$ tests.

To estimate the cumulative incidence of outcomes, taking into account the risk of death from other causes, we used the Aalen-Johansen method (competing risks). ${ }^{22}$

We used time dependent Cox proportional hazards models to examine the risk of events during follow-up for the different groups restarting antithrombotic treatment, with patients not resuming antithrombotic treatment as reference. As the number of patients who restarted triple treatment was small, we excluded them from the analysis. We constituted the different antithrombotic treatment regimens as time varying covariates, which allowed patients to switch their treatment regimen during follow-up. The models were adjusted for factors in the $\mathrm{CHA}_{2} \mathrm{DS}_{2}$-VASc score and for treatment with antiplatelets during the 90 days blanking period. We considered a two sided significance level of less than 0.05 to be significant. We checked to ensure that the Cox models all fulfilled the proportional hazard assumption and linearity of continuous covariates (age). In addition, we explored relevant interactions without any clinical significant findings, including no sex based differences.

Data management and statistical analyses were performed using SAS (version 9.2 for Windows, SAS Institute, NC) and R (version 3.0.2 for Windows, R Foundation for Statistical Computing).

\section{Patient involvement}

No patients were involved in setting the research question or the outcome measures, nor were they involved in the design and implementation of the study. There are no plans to involve patients in dissemination of the results.

\section{Results}

Index study population

Figure 1 shows the selection of the study population from 1 January 1996 to 31 December 2012. Overall, 4602

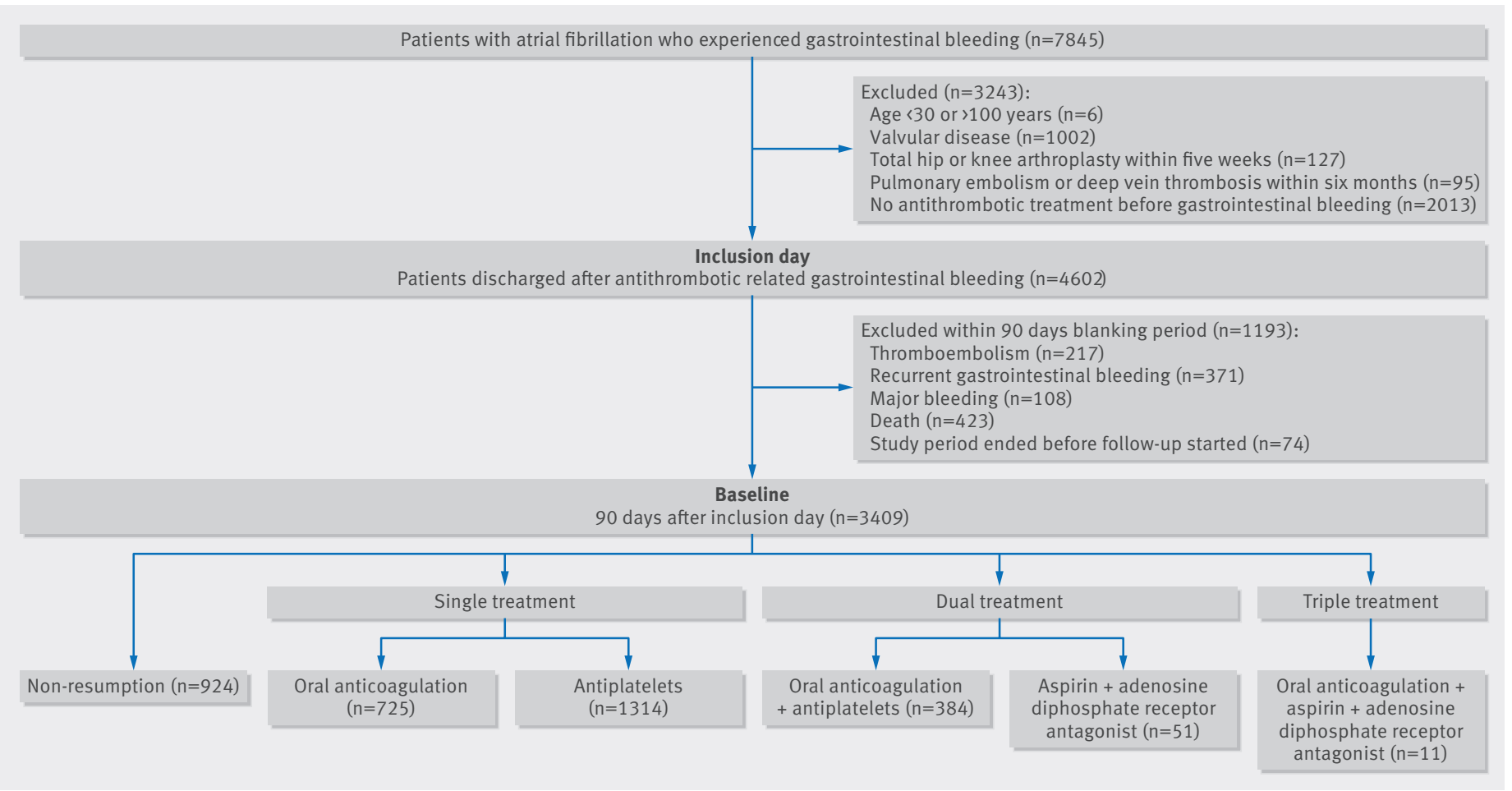

Fig 1 | Selection of study population. Antiplatelets comprise aspirin or adenosine diphosphate receptor antagonists 


\begin{tabular}{|c|c|}
\hline Characteristics & $\begin{array}{l}\text { Total population } \\
(n=4602)\end{array}$ \\
\hline Mean (SD) age, years & $78.3(9.3)$ \\
\hline Women & $2085(45.3)$ \\
\hline Mean (SD) $\mathrm{CHADS}_{2}$ score & $2.1(1.2)$ \\
\hline Mean (SD) $\mathrm{CHA}_{2} \mathrm{DS}_{2}$-VASc score & $3.6(1.5)$ \\
\hline Mean (SD) HAS-BLED score & $2.6(1.0)$ \\
\hline \multicolumn{2}{|l|}{ Antithrombotic treatment the day before the inclusion event: } \\
\hline Single: oral anticoagulation & $1101(23.9)$ \\
\hline Single: antiplatelets & $2450(53.3)$ \\
\hline Dual: oral anticoagulation+antiplatelets & $893(19.4)$ \\
\hline Dual: aspirin+adenosine diphosphate receptor antagonists & $117(2.5)$ \\
\hline Triple: oral anticoagulation+aspirin+adenosine diphosphate receptor antagonists & $41(0.9)$ \\
\hline \multicolumn{2}{|l|}{ Concomitant drugs: } \\
\hline Dipyridamole (persantin) & $199(4.3)$ \\
\hline Heparin & $20(0.4)$ \\
\hline Non-steroidal anti-inflammatory drug & $1126(24.5)$ \\
\hline Proton pump inhibitor & $693(15.1)$ \\
\hline $\mathrm{H}_{2}$ receptor antagonist & $121(2.6)$ \\
\hline \multicolumn{2}{|l|}{ Comorbidities: } \\
\hline Stroke or thromboembolism & $1034(22.5)$ \\
\hline Myocardial infarction & $681(14.8)$ \\
\hline Ischaemic heart disease & $1749(38.0)$ \\
\hline Peripheral arterial disease & $290(6.3)$ \\
\hline Vascular disease & $903(19.6)$ \\
\hline Heart failure & $1411(30.7)$ \\
\hline Hypertension & $2058(44.7)$ \\
\hline Diabetes & $743(16.2)$ \\
\hline Chronic kidney disease & $304(6.6)$ \\
\hline Liver failure & $64(1.4)$ \\
\hline Previous bleeding & $635(13.8)$ \\
\hline Alcohol misuse & 219 (4.8) \\
\hline Gastroesophageal reflux & $45(1.0)$ \\
\hline Gastric or duodenal ulcer & $302(6.6)$ \\
\hline Gastritis & $37(0.8)$ \\
\hline \multicolumn{2}{|l|}{ Invasive and surgical procedures: } \\
\hline Gastrointestinal surgery & $935(20.3)$ \\
\hline Gastroscopy & $1074(23.3)$ \\
\hline
\end{tabular}

patients with atrial fibrillation were discharged from hospital after a first time gastrointestinal bleed while receiving antithrombotic treatment, referred to as the inclusion day. Table 1 presents the characteristics of these patients.

\section{Outcomes after index bleeding event}

Figure 2 shows the cumulative incidences of outcomes after inclusion day accounting for competing risk of death. At two years the cumulative incidence of all cause mortality was $39.9 \%$ (95\% confidence interval $38.4 \%$ to $41.3 \%, \mathrm{n}=1745$ ), thromboembolism $12.0 \%$ (11.0\% to $13.0 \%, \mathrm{n}=526)$, major bleeding $17.7 \%$ (16.5\% to $18.8 \%, \mathrm{n}=788)$, or recurrent gastrointestinal bleeding $12.1 \%(11.1 \%$ to $13.1 \%, n=546)$. The incidences of all cause mortality, major bleeding, and recurrent gastrointestinal bleeding increased noticeably within the first month after the inclusion day, whereas the incidence of thromboembolism showed a regular increase over two years.

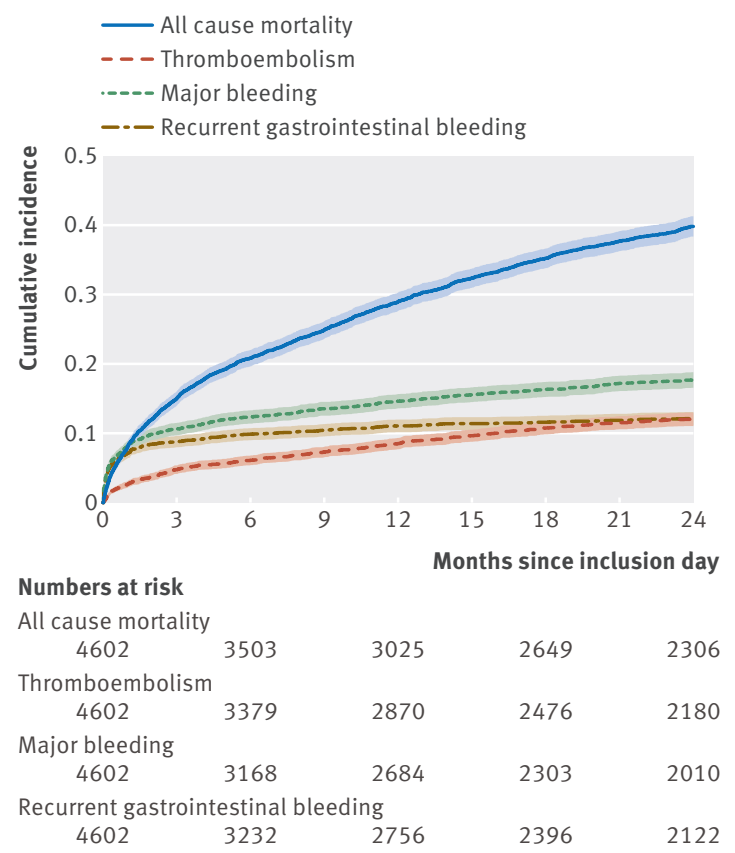

Fig 2 | Cumulative incidences of all cause mortality, thromboembolism, major bleeding, or recurrent gastrointestinal bleeding counted from day after discharge from hospital for antithrombotic related gastrointestinal bleeding. For outcomes thromboembolism, major bleeding, or recurrent gastrointestinal bleeding the model accounts for risk of death from other causes (competing risks)

\section{Baseline study population}

Overall, we excluded 1193 patients in the 90 days blanking period between the inclusion day and baseline (fig 1). The baseline study population thus comprised 3409 patients. Table 2 shows the baseline characteristics of this population.

The overall mean age of the baseline population was 77.9 (SD 9.3) years, and 44.6\% $(n=1521)$ of the patients were women. The mean $\mathrm{CHA}_{2} \mathrm{DS}_{2}$-VASc score was 3.6 (SD 1.5) and mean HAS-BLED score was 3.0 (SD 1.0).

Antithrombotic treatment was restarted in $72.9 \%$ $(n=2485)$ of the patients: single treatment with oral anticoagulation $(21.3 \%, n=725)$, aspirin $(35.5 \%$, $\mathrm{n}=1212$ ), or an adenosine diphosphate receptor antagonist (3.0\%, $\mathrm{n}=102)$; dual treatment with oral anticoagulation plus aspirin $(10.7 \%, \mathrm{n}=363)$, oral anticoagulation plus an adenosine diphosphate receptor antagonist $(0.6 \%, \mathrm{n}=21)$, or aspirin plus an adenosine diphosphate receptor antagonist $(1.5 \%, n=51)$; or triple treatment with oral anticoagulation plus aspirin plus an adenosine diphosphate receptor antagonist $(0.3 \%$, $\mathrm{n}=11$ ). Of 725 patients who restarted single treatment with oral anticoagulation, 10 patients restarted dabigatran and one rivaroxaban. Furthermore, 384 patients restarted dual treatment with oral anticoagulation plus an antiplatelet agent, of whom two received dabigatran plus an antiplatelet agent. Non-vitamin K antagonist oral anticoagulants were not included in the other baseline groups. 


$$
\bar{E}
$$



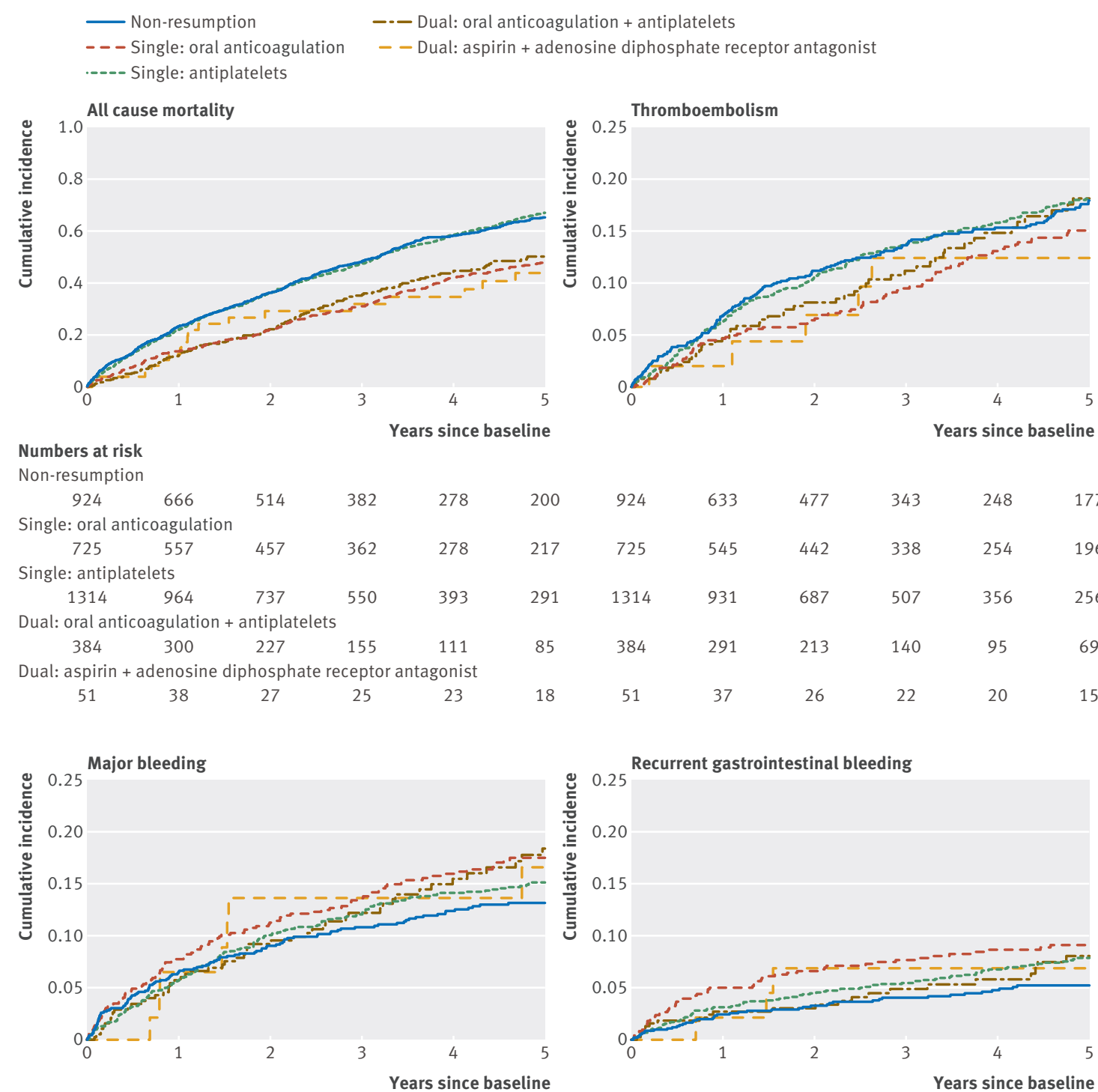

Numbers at risk

\begin{tabular}{|c|c|c|c|c|c|c|c|c|c|c|c|}
\hline \multicolumn{12}{|c|}{ Non-resumption } \\
\hline 725 & 524 & 418 & 321 & 242 & 196 & 725 & 532 & 435 & 341 & 256 & 197 \\
\hline 1314 & 925 & 677 & 489 & 336 & 256 & 1314 & 939 & 708 & 522 & 365 & 264 \\
\hline \multicolumn{12}{|c|}{ Dual: oral anticoagulation + antiplatelets } \\
\hline 384 & 286 & 205 & 135 & 92 & 69 & 384 & 290 & 219 & 146 & 104 & 77 \\
\hline 51 & 36 & 23 & 21 & 20 & 15 & 51 & 37 & 25 & 23 & 21 & 17 \\
\hline
\end{tabular}

Fig 3 | Cumulative incidences of all cause mortality, thromboembolism, major bleeding, or recurrent gastrointestinal bleeding after baseline, stratified by baseline groups. For outcomes thromboembolism, major bleeding, or recurrent gastrointestinal bleeding the model accounts for risk of death from other causes (competing risks). Antiplatelets comprise aspirin or adenosine diphosphate receptor antagonists

\section{Cumulative incidences}

Figure 3 shows cumulative incidences for all cause mortality, thromboembolism, major bleeding, or recurrent gastrointestinal bleeding stratified by baseline groups (similar to intention to treat) accounting for competing risk of death. The incidence of all cause mortality within the first five years increased significantly for non-resumption and restart of single treatment with antiplatelets compared with the other baseline groups. The cumulative incidence of thromboembolism was generally lowest for restart of oral anticoagulation; however, this was not significant in relation to the other baseline groups.

\section{Risk of outcomes associated with antithrombotic treatment after baseline}

The median duration of follow-up was 2.0 years (interquartile range 0.7 to 4.0 years). Table 3 presents the time dependent adjusted hazard ratios of outcomes with 


\begin{tabular}{|c|c|c|c|c|c|}
\hline Outcomes & $\begin{array}{l}\text { No of } \\
\text { events }\end{array}$ & \multicolumn{2}{|l|}{ Single treatment } & \multicolumn{2}{|l|}{ Dual treatment } \\
\hline All cause mortality & 1730 & $0.39(0.34$ to 0.46$)$ & 0.76 (0.68 to 0.86$)$ & $0.41(0.32$ to 0.52$)$ & $0.88(0.57$ to 1.36$)$ \\
\hline Major bleeding & 454 & 1.37 (1.06 to 1.77$)$ & $1.25(0.96$ to 1.62$)$ & 1.44 (1.00 to 2.08$)$ & $1.36(0.54$ to 3.43$)$ \\
\hline Recurrent gastrointestinal bleeding & 216 & $1.22(0.84$ to 1.77$)$ & $1.19(0.82$ to 1.74$)$ & 1.34 (0.79 to 2.28$)$ & $0.58(0.08$ to 4.30$)$ \\
\hline
\end{tabular}

antithrombotic treatment during follow-up in patients surviving the first 90 days after gastrointestinal bleeding. The reference was non-resumption of antithrombotic treatment.

Restart of single treatment with oral anticoagulation was associated with the lowest rate of all cause mortality (hazard ratio $0.39,0.34$ to 0.46 ) and thromboembolism (0.41, 0.31 to 0.54). Among the different treatment regimens that were restarted, the risk of major bleeding was significantly increased only in patients who restarted single treatment with oral anticoagulation (1.37, 1.06 to 1.77$)$; however, the risk of recurrent gastrointestinal bleeding associated with any of the restarted groups was not significantly different from the reference group.

Additional analyses stratifying the study population at the inclusion event into three groups according to $\mathrm{CHA}_{2} \mathrm{DS}_{2}$-VASc score $<2, \mathrm{CHA}_{2} \mathrm{DS}_{2}$-VASc score 2 or 3 , and $\mathrm{CHA}_{2} \mathrm{DS}_{2}$-VASc score $>3$ indicated that a gradual increase of the $\mathrm{CHA}_{2} \mathrm{DS}_{2}$-VASc score at the inclusion event was associated with a gradual decrease in the risk of all cause mortality among patients who restarted single treatment with oral anticoagulation. Moreover, a HAS-BLED score $>3$ at the inclusion event showed an increased associated risk of recurrent gastrointestinal bleeding in patients who restarted single treatment with oral anticoagulation (see supplementary table 3).

Based on the results from table 2, we performed a subgroup analysis stratifying patients into antithrombotic treatment at the inclusion event (single treatment with oral anticoagulation or an antiplatelet agent, or dual treatment with oral anticoagulation plus an antiplatelet agent). The results showed that independent of antithrombotic treatment before the gastrointestinal bleeding, restarting or modifying treatment to single treatment with oral anticoagulation was in general associated with the greatest effectiveness and a relative safety compared with other restarted treatment strate- gies and non-resumption of antithrombotic treatment (see supplementary table 4).

\section{Concomitant proton pump inhibitor use after gastrointestinal bleeding}

We carried out a subgroup analysis including patients who received concomitant treatment with proton pump inhibitors after gastrointestinal bleeding (table 4).

\section{Sensitivity analyses}

Supplementary table 2 presents the numbers and risks of events within one year of follow-up, and the results showed consistency with the main results in table 3 . Supplementary figure 1 shows cumulative incidences of events within five years after the inclusion day.

For the purpose of sensitivity analyses, we changed the 90 days blanking period to 30,60 , and 120 days. The results based on a shorter or longer blanking period supported the main results with the 90 days blanking period (table 5).

We carried out four additional sensitivity analyses. Firstly, we used unadjusted Cox models. Secondly, we adjusted the Cox models for potential confounders according to previous gastroscopy, cancer, chronic kidney disease, liver failure, alcohol misuse, and the use of non-steroidal anti-inflammatory drugs and dipyridamole 90 days before baseline. Thirdly, we carried out analyses with oral anticoagulation separated into vitamin $\mathrm{K}$ antagonists and non-vitamin $\mathrm{K}$ antagonist oral anticoagulants, and antiplatelets separated into aspirin and adenosine diphosphate receptor antagonists. Finally, we carried out analyses with follow-up starting the day after discharge (without the 90 days blanking period), assuming patients continued with the same antithrombotic treatment after discharge as before the inclusion event, and until they ran out of tablets. Supplementary table 5 shows the results of the sensitivity analyses; overall, the results were similar to the main results in table 3.

\begin{tabular}{|c|c|c|c|c|}
\hline \multirow[b]{2}{*}{ Outcomes } & \multicolumn{2}{|l|}{ Single treatment } & \multicolumn{2}{|l|}{ Dual treatment } \\
\hline & $\begin{array}{l}\text { Oral } \\
\text { anticoagulation }\end{array}$ & Antiplatelets & $\begin{array}{l}\text { Oral } \\
\text { anticoagulation+ } \\
\text { antiplatelets }\end{array}$ & $\begin{array}{l}\text { Aspirin+adenosine } \\
\text { diphosphate } \\
\text { receptor antagonist }\end{array}$ \\
\hline All cause mortality & 0.38 (0.32 to 0.45$)$ & 0.75 (0.67 to 0.85$)$ & 0.41 (0.31 to 0.53$)$ & 0.78 (0.49 to 1.24$)$ \\
\hline Thromboembolism & 0.40 (0.30 to 0.54$)$ & 0.75 (0.60 to 0.95$)$ & 0.57 (0.37 to 0.87$)$ & 0.77 (0.33 to 1.80$)$ \\
\hline Major bleeding & 1.45 (1.10 to 1.91$)$ & 1.26 (0.95 to 1.67$)$ & 1.50 (1.02 to 2.22$)$ & 1.44 (0.57 to 3.67$)$ \\
\hline Recurrent gastrointestinal bleeding & $1.26(0.85$ to 1.87$)$ & 1.09 (0.73 to 1.64$)$ & 1.30 (0.74 to 2.29$)$ & 0.58 (0.08 to 4.36$)$ \\
\hline
\end{tabular}




\begin{tabular}{|c|c|c|c|c|}
\hline \multirow[b]{2}{*}{ Blanking periods } & \multicolumn{4}{|l|}{ Outcomes } \\
\hline & All cause mortality & Thromboembolism & Major bleeding & $\begin{array}{l}\text { Recurrent } \\
\text { gastrointestinal } \\
\text { bleeding }\end{array}$ \\
\hline \multicolumn{5}{|l|}{30 days blanking period* } \\
\hline No of events & 2063 & 581 & 570 & 294 \\
\hline \multicolumn{5}{|l|}{ Single treatment: } \\
\hline Oral anticoagulation $(\mathrm{n}=783)$ & 0.39 (0.34 to 0.45$)$ & 0.45 (0.35 to 0.59$)$ & $1.31(1.02$ to 1.68$)$ & $1.22(0.86$ to 1.72$)$ \\
\hline Antiplatelets $(n=1814)$ & 0.79 (0.71 to 0.87$)$ & $0.80(0.66$ to 0.96$)$ & $1.24(1.00$ to 1.55$)$ & $1.21(0.90$ to 1.64$)$ \\
\hline \multicolumn{5}{|l|}{ Dual treatment: } \\
\hline Oral anticoagulation+antiplatelets $(n=663)$ & $0.50(0.41$ to 0.61$)$ & $0.58(0.41$ to 0.83$)$ & $1.28(0.93$ to 1.75$)$ & $1.13(0.72$ to 1.77$)$ \\
\hline Aspirin+adenosine diphosphate receptor antagonists $(n=115)$ & 0.74 (0.50 to 1.09$)$ & 0.68 (0.32 to 1.48$)$ & 2.06 (1.13 to 3.74$)$ & $1.88(0.79$ to 4.51$)$ \\
\hline \multicolumn{5}{|l|}{60 days blanking periodt } \\
\hline No of events & 1857 & 532 & 490 & 234 \\
\hline \multicolumn{5}{|l|}{ Single treatment: } \\
\hline Oral anticoagulation $(n=707)$ & $0.38(0.33$ to 0.45$)$ & $0.43(0.33$ to 0.57$)$ & $1.40(1.08$ to 1.82$)$ & 1.23 (0.84 to 1.78$)$ \\
\hline Antiplatelets $(n=1575)$ & 0.79 (0.71 to 0.88$)$ & $0.79(0.65$ to 0.97$)$ & $1.30(1.03$ to 1.64$)$ & $1.28(0.92$ to 1.78$)$ \\
\hline \multicolumn{5}{|l|}{ Dual treatment: } \\
\hline Oral anticoagulation+antiplatelets $(n=506)$ & $0.49(0.40$ to 0.61$)$ & 0.59 (0.40 to 0.86$)$ & $1.47(1.05$ to 2.06$)$ & $1.44(0.89$ to 2.33$)$ \\
\hline Aspirin+adenosine diphosphate receptor antagonists $(n=84)$ & $0.81(0.53$ to 1.22$)$ & 0.85 (0.39 to 1.83$)$ & 1.52 (0.70 to 3.30$)$ & $0.50(0.07$ to 3.60$)$ \\
\hline \multicolumn{5}{|l|}{120 days blanking period $\neq$} \\
\hline No of events & 1414 & 408 & 390 & 177 \\
\hline \multicolumn{5}{|l|}{ Single treatment: } \\
\hline Oral anticoagulation $(n=724)$ & $0.36(0.30$ to 0.43$)$ & $0.43(0.31$ to 0.58$)$ & 1.41 (1.05 to 1.88$)$ & $1.17(0.76$ to 1.79$)$ \\
\hline Antiplatelets ( $\mathrm{n}=1182)$ & $0.77(0.69$ to 0.87$)$ & $0.77(0.61$ to 0.96$)$ & $1.37(1.06$ to 1.78$)$ & 1.25 (0.86 to 1.83$)$ \\
\hline \multicolumn{5}{|l|}{ Dual treatment: } \\
\hline Oral anticoagulation+antiplatelets $(\mathrm{n}=333)$ & $0.44(0.34$ to 0.58$)$ & 0.52 (0.33 to 0.81$)$ & $1.63(1.12$ to 2.37$)$ & 1.48 (0.86 to 2.55$)$ \\
\hline Aspirin+adenosine diphosphate receptor antagonists $(n=50)$ & $0.76(0.47$ to 1.25$)$ & 0.99 (0.43 to 2.28$)$ & 1.52 (0.61 to 3.78$)$ & $0.66(0.09$ to 4.86$)$ \\
\hline
\end{tabular}

\section{Discussion}

In this nationwide study of 4602 patients with nonvalvular atrial fibrillation who were discharged from hospital after a gastrointestinal bleeding event while receiving antithrombotic treatment, we found the cumulative incidence of death was high; 39.9\% within two years. Secondly, $27.1 \%$ of the patients did not resume antithrombotic treatment after the gastrointestinal bleed. Thirdly, among patients surviving the first 90 days after gastrointestinal bleeding, restarting single treatment with oral anticoagulation was associated with the lowest risk of all cause mortality and thromboembolism compared with non-resumption of antithrombotic treatment. Fourthly, none of the restarted antithrombotic treatment regimens was associated with a significantly increased risk of recurrent gastrointestinal bleeding.

\section{Gastrointestinal bleeding as a powerful indicator of death}

Gastrointestinal bleeding is a serious event with high case fatality, and this study supports the results from the UK national audit 2007.23 Gastrointestinal bleeding is associated with a high mortality, especially among elderly patients with atrial fibrillation and multiple comorbidities who take antithrombotics, which is in accordance with our results and those of other studies. ${ }^{24}$ These findings reinforce the importance of gastrointestinal bleeding as a powerful indicator of death-that is, in our study $39.9 \%$ of patients died within two years after gastrointestinal bleeding. Stroke prevention among these high risk patients is a clinical challenge and a multidisciplinary task.

\section{Antithrombotic treatment after gastrointestinal bleeding}

The decision not to resume antithrombotic treatment might be made to avoid rebleeding at the expense of an increased risk of stroke after gastrointestinal bleeding in patients with atrial fibrillation, and our observational data showed that $27.1 \%$ of patients did not resume antithrombotic treatment after gastrointestinal bleeding. In a comparable retrospective study, Qureshi and colleagues ${ }^{9}$ reported that $50.9 \%$ of patients with atrial fibrillation did not resume warfarin treatment after gastrointestinal bleeding. In the present study it was an option for patients to restart single treatment with antiplatelets, and we found that a large proportion started this treatment (aspirin $35.5 \%$ and adenosine diphosphate receptor antagonist $3.0 \%$ ) with a mean $\mathrm{CHA}_{2} \mathrm{DS}_{2}$-VASc score of 3.7 (SD 1.5), despite that it has been established that patients with atrial fibrillation have the most net-clinical benefit from single treatment with oral anticoagulation. ${ }^{1162526}$ This is further supported by our study, which suggests that after gastrointestinal bleeding the benefits of restarting oral anticoagulation outweigh the risks of recurrent bleeding compared with other antithrombotic treatment regimens or non-resumption of antithrombotics-that is, single treatment with oral 
anticoagulation reduced mortality. Furthermore, this is in agreement with the study by Qureshi and colleagues, who reported benefit of restarting warfarin on mortality and thromboembolism and without the additional cost of recurrent gastrointestinal bleeding. ${ }^{9}$ Similarly, Witt and colleagues ${ }^{27}$ found that after gastrointestinal bleeding, restarting warfarin treatment compared with no treatment was associated with a lower risk of death and thromboembolism without a significantly increased risk of recurrent gastrointestinal bleeding. However, this study did not focus solely on patients with atrial fibrillation. A randomised controlled trial by Sung and colleagues ${ }^{28}$ tested continuation of single treatment with aspirin compared with no treatment after ulcer related bleeding. Despite the study's small sample size of 156 patients initially treated with aspirin as prophylaxis for cardiovascular or cerebrovascular diseases, the authors concluded that continuing aspirin treatment versus non-resumption potentially reduced mortality but increased the risk of recurrent gastrointestinal bleeding. The results of our observational study draw a parallel to those of that study, that restarting treatment is beneficial and outweighs the risks of recurrent bleeding; however, our study exclusively included patients with atrial fibrillation, and they benefited most from restarting single treatment with oral anticoagulation.

In the current study, patients who restarted single treatment with antiplatelets or combination treatment had the highest risk scores of stroke and bleeding, as indicated by the $\mathrm{CHA}_{2} \mathrm{DS}_{2}$-VASc score and HAS-BLED score, respectively. Even though we adjusted for these risk scores, confounding by indication may have influenced our results leading to an underestimation of subsequent stroke and an overestimation of recurrent bleeding in these treatment groups. However, this study came across a surprising finding-that patients who restarted single treatment with oral anticoagulation and those who did not resume antithrombotic treatment had similar risk scores for stroke $\left(\mathrm{CHA}_{2} \mathrm{DS}_{2}\right.$-VASc: 3.3 (SD 1.6) and 3.4 (SD 1.5), respectively) and bleeding (HAS-BLED: 2.6 (SD 0.9) and 2.6 (SD 0.9), respectively), but during follow-up patients who restarted single treatment with oral anticoagulation benefited from a decreased associated risk of all cause mortality and thromboembolism without an increased risk of recurrent gastrointestinal bleeding. This suggests the need and importance of restarting single treatment with oral anticoagulation in patients with atrial fibrillation after gastrointestinal bleeding.

\section{The blanking period}

During the 90 days blanking period, we missed a proportion of events. Moreover, the results could be less generalisable in a clinical every day practice, because the 90 days blanking period obscured what happened in terms of antithrombotic treatment within the first 90 days after the gastrointestinal bleeding. To evaluate the effect of changing the blanking period, we carried out sensitivity analyses not including the blanking period or changing the blanking period to 30, 60, or 120 days. The results from the sensitivity analyses supported the main results based on the 90 days blanking period.

\section{Use of proton pump inhibitors after gastrointestinal} bleeding

In international guidelines the use of proton pump inhibitors is indicated in patients with a history of gastrointestinal bleeding treated with dual antiplatelet drugs or concomitant anticoagulation treatment. ${ }^{2930}$ In this study, the use of proton pump inhibitors after gastrointestinal bleeding in the groups who restarted antithrombotic treatment ranged from $90.0 \%$ to $96.1 \%$ at baseline, showing that recommendations are generally followed. The results from the subgroup analysis based on patients who restarted antithrombotic treatment and used concomitant proton pump inhibitors did not deviate much from the main results (table 4).

\section{Restart of antithrombotic treatment and recurrent bleeding}

One of our objectives was to examine major bleeding, which to our knowledge has not been examined previously in the selected study population. In patients with atrial fibrillation, combination treatment is associated with a higher risk of major bleeding than is single treatment. ${ }^{4}$ Moreover, drug-drug interactions between antithrombotics increase the risk of gastrointestinal bleeding, and Delaney and colleagues ${ }^{31}$ concluded that dual treatment with aspirin added to warfarin or clopidogrel was associated with a greater risk of gastrointestinal bleeding than that observed with each drug alone. The present study found that single or dual treatment was not significantly associated with an increased risk of recurrent gastrointestinal bleeding; however, lack of statistical power could have influenced this result.

\section{Non-vitamin K antagonist oral anticoagulants after gastrointestinal bleeding}

In the past three or four years, the use of non-vitamin $\mathrm{K}$ antagonist oral anticoagulants is increasing at the expense of vitamin $\mathrm{K}$ antagonists, ${ }^{21}$ but owing to the paucity of data on non-vitamin $\mathrm{K}$ antagonist oral anticoagulants in the current study we cannot conclude anything on the efficacy or safety of these drugs in this setting. Consideration of the patient's profile is necessary when balancing the risks of stroke with recurrent bleeding after gastrointestinal bleeding in patients with atrial fibrillation.

\section{Strengths and limitations of this study}

The major strength of the present study was the opportunity to include a nationwide cohort of 4602 patients with atrial fibrillation independent of sex, age, socioeconomic status, or participation in health insurance programmes. All Danish residents are covered by a public health insurance system, which ensures registration of all hospital admissions and outpatient contacts. In addition, pharmacies register all claimed 
prescriptions, and the Danish registries are well validated. ${ }^{123233}$ Aspirin, proton pump inhibitors, non-steroidal anti-inflammatory drugs, and $\mathrm{H}_{2}$ receptor antagonists are also available over the counter; however, owing to a partial reimbursement system in Denmark, chronic users mainly claim medicine on prescription. In 2012, 92\% of all aspirin was sold as a prescription drug. ${ }^{34}$ The time dependent statistical models based on all prescriptions claimed added strength to the results, but from the registries we were not able to determine whether patients took their tablets after the prescription had been dispensed. This could have led to a potential overestimation of events in the groups who restarted antithrombotic drugs. Another limitation was that patients included near the end of the study had decreased risk time to develop an event. We used the major and non-major diagnosis codes of gastrointestinal bleeding to ensure high sensitivity, but no detailed clinical information was available for any of the events. We were not able to distinguish between lower and upper gastrointestinal bleeding, owing to lack of power.

The international normalised ratio levels, the quality of warfarin control, serum creatinine concentration, renal function, haemoglobin levels, and other potential confounders with no possibilities for identification in the registries, could have influenced our results. We attempted to minimise these influences by carrying out sensitivity analysis-for example, by adjusting for chronic kidney disease and by using methods from previous parallel studies. ${ }^{4} 1516$

The observational study design did not permit definite causations to be concluded; instead, we examined the associations between restart of antithrombotic treatment and outcomes. The demand for a randomised controlled trial on restarting oral anticoagulation and the risk of stroke and recurrent bleeding after a gastrointestinal bleeding among patients with atrial fibrillation is increasing, but such a trial would face many challenges.

\section{Conclusion}

Mortality was high among patients with atrial fibrillation who were discharged from hospital after a gastrointestinal bleeding event while taking antithrombotics. More than a quarter did not resume treatment. However, among patients surviving the first 90 days after gastrointestinal bleeding, restarting single treatment with oral anticoagulation was associated with the lowest risk of all cause mortality and thromboembolism, and a relative safe use regarding the risk of recurrent bleeding, compared with non-resumption of antithrombotic treatment or restarting other antithrombotic treatment regimens.

Contributors: GYHL and GHG are joint senior authors. LS, GYHL, JBO, and $\mathrm{GHG}$ conceived and designed the study and carried out the statistical analysis. GYHL and GHG provided administrative, technical, or material support. GYHL, JBO, ELF, JLP, AG, ANB, TBL, CT-P, and GHG supervised the study. LS drafted the manuscript. All authors acquired, analysed, or interpreted the data, critically revised the manuscript for important intellectual content, and gave final approval of the version for publication. LS and GHG had full access to all of the data in the study and take responsibility for the integrity of the data, for the accuracy of the data analysis, and that the manuscript is an honest, accurate, and transparent account of the study. LS is the guarantor.

Funding: This study was supported by a grant from BoehringerIngelheim. The sponsor had no influence on the study design, interpretation of results, or the decision to submit the manuscript for publication.

Competing interests: All authors have completed the ICMJE uniform disclosure form at www.icmje.org/coi disclosure.pdf and declare: GYHL has served as a consultant for Bayer, Astellas, Merck, Sanofi, Pfizer/Bristol Meyers Squibb, Daiichi-Sankyo, Biotronik, Medtronic, Portola, and Boehringer Ingelheim and has been on the speakers' bureau for Bayer, Pfizer/Bristol Meyers Squibb, Boehringer Ingelheim, Daiichi-Sankyo, Medtronic, and Sanofi Aventis. JBO has received speaker fees from Bristol Myers Squibb and Boehringer Ingelheim and funding for research from the Lundbeck Foundation, Bristol-Myers Squibb, and the Capital Region of Denmark, Foundation for Health Research. ELF was previously supported by a project specific research grant from Janssen Pharmaceuticals and has received funding for research from the Lundbeck Foundation and Bristol-Myers Squibb. AG has received funding from Bristol-Myers Squibb. GHG has received research grants from Pfizer/Bristol Meyers Squibb, Bayer, BoehringerIngelheim, and AstraZeneca and speaker fees from AstraZeneca, Pfizer, and Sanofi Aventis. All authors state independence from the funders.

Ethical approval: Retrospective registry based studies do not require approval from the research ethics committee system in Denmark; data were structured with no possibility for individual patient identification but allowed linkage between registries. The Danish Data Protection Agency had approved use of data for the study (reference No 2007-58-0015/GEH-2014-012 I-Suite No 02720)

Data sharing: No additional data available. Details of statistical analysis are available from the corresponding author (Lailastaerk@ gmail.com) on request.

Transparency: The lead author (LS) affirms that the manuscript is an honest, accurate, and transparent account of the study being reported; that no important aspects of the study have been omitted; and that any discrepancies from the study as planned have been explained.

This is an Open Access article distributed in accordance with the Creative Commons Attribution Non Commercial (CC BY-NC 4.0) license, which permits others to distribute, remix, adapt, build upon this work non-commercially, and license their derivative works on different terms, provided the original work is properly cited and the use is non-commercial. See: http://creativecommons.org/licenses/ by-nc/4.0/.

1 Lip GH, Lane DA. Stroke prevention in atrial fibrillation: a systematic review. JAMA 2015:313:1950-62.

2 Camm AJ, Lip GYH, Caterina RD, et al. 2012 focused update of the ESC guidelines for the management of atrial fibrillation: an update of the 2010 ESC guidelines for the management of atrial fibrillationdeveloped with the special contribution of the European Heart Rhythm Association. Europace 2012;14:1385-413.

3 Sam C, Massaro JM, D’Agostino RB Sr, et al. Warfarin and aspirin use and the predictors of major bleeding complications in atrial fibrillation (the Framingham Heart Study). Am J Cardiol 2004;94:947-51.

4 Hansen ML, Sørensen R, Clausen MT, et al. Risk of bleeding with single, dual, or triple therapy with warfarin, aspirin, and clopidogrel in patients with atrial fibrillation. Arch Intern Med 2010;170:1433-41.

5 Choudari CP, Palmer KR. Acute gastrointestinal haemorrhage in patients treated with anticoagulant drugs. Gut 1995;36:483-4

6 Man-Son-Hing M, Laupacis A. Balancing the risks of stroke and upper gastrointestinal tract bleeding in older patients with atrial fibrillation. Arch Intern Med 2002;162:541-50.

7 Camm Al, Kirchhof P, Lip GYH et al. Guidelines for the management of atrial fibrillation. The Task Force for the Management of Atria Fibrillation of the European Society of Cardiology (ESC). Europace 2010;12:1360-420.

8 Alberts MJ, Eikelboom JW, Hankey GJ. Antithrombotic therapy for stroke prevention in non-valvular atrial fibrillation. Lancet Neurol 2012;11:1066-81.

9 Qureshi W, Mittal C, Patsias I, et al Restarting anticoagulation and outcomes after major gastrointestinal bleeding in atrial fibrillation. Am J Cardiol 2014;113:662-8

10 Riva N, Apostolakis S, Lip GYH. Oral antithrombotic therapy and gastrointestinal bleeding: the good, the bad and the ugly. Int J Clin Pract 2012;66:2-4

11 Lynge E, Sandegaard JL, Rebolj M. The Danish National Patient Register. Scand I Public Health 2011:39:30-3.

12 Kildemoes HW, Sørensen HT, Hallas J. The Danish National Prescription Registry. Scand J Public Health 2011;39:38-41

13 Pedersen CB. The Danish Civil Registration System. Scand J Public Health 2011:39:22-5. 
14 Cunningham A, Stein CM, Chung CP, et al. An automated database case definition for serious bleeding related to oral anticoagulant use. Pharmacoepidemiol Drug Saf 2011:20:560-6.

15 Lamberts M, Olesen JB, Ruwald MH, et al. Bleeding after initiation of multiple antithrombotic drugs, including triple therapy, in atrial fibrillation patients following myocardial infarction and coronary intervention: a nationwide cohort study. Circulation 2012;126:1185-93.

16 Olesen JB, Lip GYH, Lindhardsen J, et al. Risks of thromboembolism and bleeding with thromboprophylaxis in patients with atrial fibrillation: a net clinical benefit analysis using 'real world' nationwide cohort study. Thromb Haemost 2011;106:739-49.

17 Olesen IB, Lip GYH, Hansen ML et al Validation of risk stratification schemes for predicting stroke and thromboembolism in patients with atrial fibrillation: nationwide cohort study. BMJ 2011;342:d124.

18 Kümler T, Gislason GH, Kirk V, et al. Accuracy of a heart failure diagnosis in administrative registers. Eur J Heart Fail 2008;10:658-60.

19 Krarup L-H, Boysen G, Janjua H, et al. Validity of stroke diagnoses in a national register of patients. Neuroepidemiology 2007;28:150-4.

20 Madsen M, Davidsen M, Rasmussen S, et al. The validity of the diagnosis of acute myocardial infarction in routine statistics: a comparison of mortality and hospital discharge data with the Danish MONICA registry. J Clin Epidemiol 2003;56:124-30

21 Olesen JB, Sørensen R, Hansen ML, et al. Non-vitamin K antagonist oral anticoagulation agents in anticoagulant naïve atrial fibrillation patients: Danish nationwide descriptive data 2011-2013. Europace 2015;17:187-93.

22 Gooley TA, Leisenring W, Crowley J, et al. Estimation of failure probabilities in the presence of competing risks: new representations of old estimators. Stat Med 1999;18:695-706.

23 Hearnshaw SA, Logan RFA, Lowe D, et al. Acute upper gastrointestinal bleeding in the UK: patient characteristics, diagnoses and outcomes in the 2007 UK audit. Gut 2011;60:1327-35.

24 Lau JY, Barkun A, Fan D, et al. Challenges in the management of acute peptic ulcer bleeding. Lancet 2013;381:2033-43.

25 Lip GYH. Don't add aspirin for associated stable vascular disease in a patient with atrial fibrillation receiving anticoagulation. BM 2008;336:614-5.

26 Connolly SJ, Ezekowitz MD, Yusuf S, et al. Dabigatran versus warfarin in patients with atrial fibrillation. N Engl J Med 2009;361:1139-51.
27 Witt DM, Delate T, Garcia DA, et al. Risk of thromboembolism, recurrent hemorrhage, and death after warfarin therapy interruption for gastrointestinal tract bleeding. Arch Intern Med 2012;172:1484-91.

28 Sung JJY, Lau JYW, Ching JYL, et al. Continuation of low-dose aspirin therapy in peptic ulcer bleeding: a randomized trial. Ann Intern Med 2010;152:1-9.

29 Bhatt DL, Scheiman J, Abraham NS, et al. ACCF/ACG/AHA 2008 expert consensus document on reducing the gastrointestinal risks of antiplatelet therapy and NSAID use: a report of the American College of Cardiology Foundation Task Force on clinical expert consensus documents. J Am Coll Cardiol 2008;52:1502-17.

30 Abraham NS, Hlatky MA, Antman EM, et al. ACCF/ACG/AHA 2010 expert consensus document on the concomitant use of proton pump inhibitors and thienopyridines: a focused update of the ACCF/ACG/ AHA 2008 expert consensus document on reducing the gastrointestinal risks of antiplatelet therapy and NSAID use. J Am Coll Cardiol 2010;56:2051-66.

31 Delaney JA, Opatrny L, Brophy JM, et al. Drug-drug interactions between antithrombotic medications and the risk of gastrointestinal bleeding. Can Med Assoc J 2007;177:347-51.

32 Gaist D, Andersen M, Aarup A-L, et al. Use of sumatriptan in Denmark in 1994-5: an epidemiological analysis of nationwide prescription data. BrJ Clin Pharmacol 1997; 43:429-33.

33 Mukamal KJ, Tolstrup JS, Friberg J, et al. Alcohol consumption and risk of atrial fibrillation in men and women: the Copenhagen City Heart Study. Circulation 2005;112:1736-42.

34 Schmidt M, Hallas J, Friis S. Potential of prescription registries to capture individual-level use of aspirin and other nonsteroidal anti-inflammatory drugs in Denmark: trends in utilization 1999-2012. Clin Epidemiol 2014;6:155-68

Supplementary information: tables showing diagnoses, invasive procedures, and drugs used to define study population, and sensitivity analyses, and figure showing cumulative incidences of events 90 days after gastrointestinal bleeding 